\title{
À la frontière entre simultanéité et contraste - traduction de marqueurs de simultanéité, de contraste et de concession
}

\author{
Maria Svensson (Université d'Uppsala, Suède)
}

\begin{abstract}
This article examines the hypothesis that the use of these French markers alors que and tandis que is more generalized in an adversative and concessive context than the use of the Swedish markers medan et samtidigt som, similar to the French markers concerning their syntactic function and semantic value of simultaneity. The aim of the study is to identify different tendencies in the translation of the French markers alors que and tandis que and the Swedish markers medan and samtidigt som, which can all be considered to be ambiguous and have a double function. Their temporal signification of simultaneity has developed in an adversative or a concessive use. Our hypothesis is however that these markers are at different stages in the procedure of grammaticalisation, and that the French markers can more easily occur in a purely adversative or concessive context.

This study, based on the occurrences of these markers in two bidirectional corpora including texts of specialist literature, ParaFraSe-HumSam (Svensson 2010), and novels, Corpus Parallèle Suédois-Français : version 2010 (Andersson 2007 ; Hellqvist 2015), shows a tendency to make explicit the adversative or concessive relation when translating the French markers tandis que and alors que, especially in the case of alors que. A difference in the use of verb tense in the clauses linked by the French and the Swedish conjunctions can also be confirmed; the French conjunctions sometimes link clauses in different verb tenses, which is rare in the case of the Swedish conjunctions medan et samtidigt som. This difference could indicate different levels of grammaticalisation of these conjunctions.
\end{abstract}

Keywords: textual markers, concession, contrast, explicitation, grammaticalisation

\section{Introduction}

Notre étude portera sur alors que et tandis que en français ainsi que sur medan et samtidigt som en suédois. Ces marqueurs se ressemblent au niveau syntaxique, étant donné qu'ils sont tous des conjonctions de subordination, et au niveau sémantique, puisqu'ils peuvent marquer la simultanéité ou la concomitance (1).

(1) Un matin, trois semaines avant Noël, alors que je revenais des courses avec un cabas bourré de navets et de mou pour le chat, je trouvai Lucien habillé, prêt pour sortir. (Barbery) 
Ils peuvent également relier deux éléments qui expriment des faits entre lesquels il y a un contraste (2), voire, dans certains cas, une relation de concession (3).

(2) Et ce n'est pas un hasard si les nouveaux mouvements sociaux sont pour la première fois largement animés par des femmes, tandis que le mouvement ouvrier et même les mouvements de libération nationale furent très largement dirigés par des hommes. (Touraine)

(3) Il nous porte et nous crucifie, en nous conduisant chaque jour au champ de bataille où nous avons perdu la veille mais qui, dans le soleil, nous semble à nouveau un terrain de conquêtes, nous fait bâtir, alors que nous mourrons demain, des empires voués à devenir poussière, comme si le savoir que nous avons de leur chute prochaine n'importait pas à la soif de les édifier maintenant [...]. (Barbery)

Dans (2), il y une mise en contraste entre deux paires d'entités, les nouveaux mouvements sociaux et le mouvement ouvrier et même les mouvements de libération nationale, ainsi qu'entre femmes et hommes, ce qui caractérise la relation adversative (voir Stage 1991, 184-187). Dans (3), il s'agit d'un obstacle surmontable, ou d'une cause qui n'est pas suivie de l'effet attendu (voir par exemple Morel 1996, 5). Le fait que nous mourrons demain, exprimé dans la subordonnée, pourrait faire croire que nous ne bâtirions plus rien, ce qui est contesté dans la proposition principale. Il s'agit donc d'une relation concessive.

Les marqueurs correspondants suédois ont le même type d'emploi, comme nous pouvons le constater dans La Grammaire de l'Académie Suédoise (Teleman, Hellberg et Andersson 1999), mais il nous semble que cet emploi est moins répandu que celui de alors que et de tandis que, ce qui nous a encouragée à comparer ces marqueurs entre les deux langues et à examiner leur traduction.

Notre objectif est d'identifier des tendances de la traduction des marqueurs à valeur double de simultanéité et de contraste dans la traduction du français vers le suédois. Notre intérêt portera particulièrement sur les relations adversative et concessive possiblement établies entre les deux faits reliés par ces marqueurs. Cette relation a-t-elle tendance à être explicitée dans la traduction? Et si c'est le cas, cette tendance dépendrait-elle du contexte ?

\section{Les marqueurs de simultanéité selon les dictionnaires et les grammaires}

Comme point de départ de notre étude, nous proposons d'examiner leur description dans les principaux dictionnaires français et suédois. Ainsi, Le Nouveau Petit Robert évoque pour tandis que les emplois temporel et adversatif. Selon le même dictionnaire, alors que véhicule quatre valeurs différentes: temporelle, adversative, comparative et une décrite comme «[e]n dépit du fait que, contrairement au fait que $\rightarrow$ bien (que)». C'est uniquement pour alors que que Le Nouveau Petit Robert renvoie à un marqueur plutôt concessif, bien que. Ni Le Grand Robert ni Larousse ne décrivent ni ne renvoient à un emploi concessif pour aucune des deux conjonctions susmentionnées, mais traitent seulement leur emploi adversatif. La première attestation de l'emploi adversatif de tandis que date, selon Le Grand Robert et le Dictionnaire historique de la langue française de Rey (1998), de 1623, et celle de cet emploi de alors que de 1422.

Les marqueurs suédois sont, quant à eux, pourvus de propriétés semblables à celles des marqueurs français. Selon le Dictionnaire de l'encyclopédie nationale 
(Nationalencyclopediens ordbok), medan est souvent employé pour exprimer un contraste ${ }^{1}$. Le Dictionnaire de l'Académie suédoise (SAOB 1943) cite un exemple datant de 1644 de l'emploi adversatif de medan, et il fournit également un exemple de 1673 de l'emploi concessif, tout en signalant que ce dernier est archaïque. En revanche, cet emploi de samtidigt som n'est pas mentionné dans le $S A O B$, mais figure dans le Dictionnaire de l'encyclopédie nationale, sous l'entrée de l'adverbe samtidigt ('en même temps'), où l'on indique que la valeur temporelle peut être affaiblie ${ }^{3}$.

C'est surtout La Grammaire de l'Académie Suédoise (Teleman et al. 1999, 601) qui s'est approfondie dans ces emplois de medan et de samtidigt som, affirmant qu'ils peuvent indiquer entre deux faits une relation de contraste, et que celle-ci peut être explicitement marquée par un adverbe de phrase comme däremot ou å andra sidan. Selon Teleman et al., cette signification de contraste est conventionnalisée ; la preuve en serait le fait que l'action de la subordonnée n'est pas nécessairement simultanée à celle de la principale, comme dans l'exemple (4) :

(4) AIK tog hem pokalen i år, medan Degerfors vann i fjol. (ibid.)

'AIK a remporté la victoire cette année, tandis que Degerfors l'a remportée l'année dernière.'

Selon Teleman et al., la conjonction samtidigt som peut également indiquer un obstacle surmontable ou une prémisse trompeuse. Cette relation, qui est donc concessive, peut être soulignée par un adverbe de phrase comme ändå, $i$ alla fall, likväl dans la subordonnée :

(5) Boken är teoretiskt spännande, samtidigt som den (ändå) är lättläst. (ibid.)

'Le livre est passionnant du point de vue théorique, alors qu'il est (quand même) facile à lire.'

Selon Teleman et al., seul samtidigt som peut marquer aussi bien le contraste que la concession, alors que medan ne peut marquer que le contraste. Cette différence semble correspondre à celle entre alors que et tandis que tels qu'ils sont décrits par Le Nouveau Petit Robert.

\section{Grammaticalisation et conjonctions de simultanéité}

En linguistique française, alors que et tandis que ont surtout été traités dans une perspective historique, d'abord par Bat-Zeev Shyldkrot (1987), et ensuite par Marchello-Nizia (2006, 129). Dans son ouvrage sur la grammaticalisation et le changement linguistique, cette dernière mentionne alors que comme exemple de marqueurs temporels qui ont développé une valeur logique, montrant qu'il peut marquer, « outre la concomitance, l'opposition ou la concession » :

(6) Il a téléphoné alors que je lui avais ordonné de ne pas le faire. (ibid.)

(7) Je suis venu à pied alors qu'il pleuvait. (ibid.)

En ce qui concerne le processus de grammaticalisation, il est divisé, selon Heine (2002), en quatre stades :

\footnotetext{
${ }^{1}$ http://www.ne.se/ordb\%C3\%B6cker/\#/sok/ne-ordbok-sv-sv?q=medan

2 http://www.saob.se/artikel/?seek=medan\&pz=1

3 http://www.ne.se/ordb\%C3\%B6cker/\#/sok/ne-ordbok-sv-sv?q=samtidigt
} 
Stage 1: there is an expression with a "normal" or source meaning occurring in different contexts

Stage 2: there is a bridging context giving rise to an inference to the effect that, rather than the source meaning, there is another meaning, the target meaning, offering a more plausible interpretation of the utterance concerned.

Stage 3: there is a new type of context, the switch context, that no longer allows for an interpretation in terms of the source meaning.

Stage 4: conventionalization (Heine 2002, 85-86)

Marchello-Nizia $(2006,258)$ appelle ces stades stade initial, stade de contexte de transition, stade de contexte de passage et stade de conventionalisation.

Les descriptions antérieures de ces conjonctions, proposées par les dictionnaires et les grammaires, ainsi que l'impression que les marqueurs suédois de concomitance sont dans une moindre mesure employés pour marquer le contraste ou la concession que les marqueurs français, nous ont menée à baser la présente étude sur les hypothèses suivantes.

Nous supposons que la théorie de grammaticalisation et l'idée de différents stades de grammaticalisation peuvent expliquer d'éventuelles tendances d'explicitation dans la traduction, et que medan et samtidigt som n'ont pas d'emplois parfaitement similaires avec leurs correspondants français alors que et tandis que, qui, eux, connaissent des emplois adversatif et concessif plus généralisés. Même si dans les deux langues, ces marqueurs se trouvent dans un passage de valeur temporelle à une valeur logique dans le processus de grammaticalisation, alors que et tandis que se situent, supposons-nous, dans un stade plus avancé dans ce processus que ne le font medan et samtidigt som, et l'emploi adversatif ou concessif de medan et samtidigt som est peut-être plus contraint que celui des marqueurs français alors que et tandis que.

Si la relation adversative ou concessive est explicitée lors de la traduction de alors que et de tandis que, il est possible que cela soit dû au fait que les marqueurs suédois ne sont pas entièrement conventionnalisés comme marqueurs de contraste ou de concession, et qu'ils ne peuvent pas être employés dans des contextes incompatibles avec la valeur source de concomitance. Même si Teleman et al. $(1999,601)$ affirme que les deux actions ne sont pas nécessairement simultanées dans leur emploi adversatif et concessif, et que la valeur de contraste des deux marqueurs et celle de concession de samtidigt som est conventionnalisée, le fait, également mentionné par Teleman et al., que l'on ajoute souvent un adverbe de phrase pour souligner ces relations pourrait à notre avis indiquer qu'ils ne le sont peut-être pas malgré tout.

Un indice d'une différence de conventionnalisation de ces emplois entre les deux langues pourrait être une tendance à explicitation du lien adversatif ou concessif lors de la traduction des marqueurs français de simultanéité. C'est pourquoi nous examinerons si la relation adversative ou concessive a tendance à être explicitée lors de la traduction de alors que et tandis que en suédois. 


\section{Corpus}

Cette étude est basée sur deux corpus bidirectionnels : le corpus ParaFraSe-HumSam, constitué de livres de littérature spécialisée (Svensson 2010) et une partie du Corpus Parallèle Suédois-Français : version 2010 (Hellqvist 2015), composé de romans. Les ouvrages du premier corpus ont été publiés entre 1986 et 2001 et ceux du deuxième entre 1991 et 2009. Les ouvrages des deux corpus ont été rédigés par des auteurs différents, et traduits par différents traducteurs.

Nous avons pu relever au total 220 occurrences de alors que et 184 occurrences de tandis que dans les textes source français des deux corpus, comme le montre tableau 1.

\begin{tabular}{|lrr|} 
& $\begin{array}{r}\text { Textes source } \\
\text { français }\end{array}$ & $\begin{array}{r}\text { Textes cible } \\
\text { français }\end{array}$ \\
\hline alors que & 220 & 108 \\
\hline tandis que & 184 & 172 \\
Total & $\mathbf{4 0 4}$ & $\mathbf{2 8 0}$
\end{tabular}

Tableau 1 : Nombre d'occurrences de alors que et de tandis que dans les textes source et les textes cible français du corpus de romans et du corpus de littérature spécialisée.

L'analyse sera donc basée sur l'ensemble des 404 occurrences de ces deux marqueurs. Les textes cible français des deux corpus comportent moins d'occurrences que les textes source. Nous pouvons également constater que, par rapport à la distribution interne des occurrences de alors que et de tandis que dans les textes source français, où ces deux marqueurs sont employés avec à peu près la même fréquence, leur distribution est différente dans les textes cible français, où l'emploi de tandis que semble être privilégié par rapport à celui de alors que. Cette différence pourrait indiquer une tendance à éviter l'emploi de alors que lors de la traduction du suédois vers le français.

La fréquence des marqueurs de simultanéité suédois dans les corpus est exposée dans tableau 2.

\begin{tabular}{|c|c|c|}
\hline & $\begin{array}{r}\text { Textes source } \\
\text { suédois }\end{array}$ & $\begin{array}{r}\text { Textes cible } \\
\text { suédois }\end{array}$ \\
\hline medan & 287 & 317 \\
\hline samtidigt som & 71 & 116 \\
\hline Total & 358 & 433 \\
\hline
\end{tabular}

Tableau 2 : Nombre d'occurrences de medan et de samtidigt som dans les textes source et les textes cible suédois du corpus de romans et du corpus de littérature spécialisée.

Par rapport aux marqueurs français, qui sont plus fréquents dans les textes source que dans les textes cible français, nous constatons un rapport contraire pour les marqueurs suédois. Il y a plus d'occurrences de ces marqueurs suédois dans les textes cible que dans les textes source suédois. 


\section{Analyse de correspondants de traduction}

L'analyse de correspondants de traduction, dont les résultats sont exposés dans tableau 3, montre que alors que est dans 50 cas traduit par medan et dans 14 occurrences par samtidigt (som) et que tandis que est traduit par medan dans 91 cas et par samtidigt (som) dans 14 cas.

\begin{tabular}{|lcr|}
\hline medan & alors que & tandis que \\
\hline samtidigt (som) & 50 & 91 \\
\hline när/då & 14 & 14 \\
\hline Autre marqueur temporel & 35 & 11 \\
\hline Marqueur adversatif & 11 & 5 \\
\hline Marqueur concessif & 28 & 32 \\
\hline Marqueur causal & 53 & 8 \\
\hline Marqueur additif & 6 & 13 \\
\hline Autres & 5 & 3 \\
\hline Non traduit & 4 & 7 \\
\hline Total & 14 & $\mathbf{1 8 4}$
\end{tabular}

Tableau 3 : Correspondants de traductions des occurrences de alors que et de tandis que des textes source français traduits en suédois.

Parmi ces cas, il y a aussi bien des cas où la relation entre l'action exprimée par la proposition principale et celle de la subordonnée est clairement temporelle, comme dans (8), que des cas où la relation est plutôt adversative, comme dans (9) :

(8) Cet homme au visage ridé, elle l'avait vu, caché derrière une table, tandis que les balles balayaient le rez-de-chaussée.

Där var mannen med rynkigt ansikte som hon senare sett sitta gömd bakom ett bord medan kulorna ven omkring honom på bottenvåningen. (Rambach)

(9) Les seconds croient tandis que les premiers savent qu'ils imaginent.

De senare tror, medan de förra vet att de fantiserar. (Muchembled)

Le cas de alors que est bien particulier. C'est le marqueur dont plusieurs occurrences ne sont pas traduites par medan ou samtidigt (som). Nous pouvons constater que des marqueurs clairement adversatifs, tels que däremot, sont employés pour traduire alors que dans 28 cas et tandis que dans 32 cas, dont (10).

(10) «Mon emmerdeuse de sœur est une petite personne intolérante et neurasthénique qui déteste les autres et qui préférerait habiter dans un cimetière où tout le monde est mort — tandis que moi, je suis une nature ouverte, joyeuse et pleine de vie. » 
"Min urjobbiga syrra är en liten intolerant och deprimerad människa som avskyr andra och helst skulle bo på en kyrkogård där alla är döda - jag däremot är öppen, gladlynt och full av liv." (Barbery)

Des marqueurs concessifs, comme fast, sont également employés, mais bien plus rarement dans le cas de tandis que (8 occurrences) que dans celui de alors que (53 occurrences, dont (11)).

(11) Il retarde de plus en plus le moment de se rendre à l'église, alors que le carillon appelant à la messe de onze heures a sonné depuis longtemps.

Han skjuter mer och mer på ögonblicket då han ska gå till kyrkan, fast klockorna har ringt till elvamässan en bra stund redan. (Rouaud)

Ceci confirme les descriptions d'alors que et de tandis que des dictionnaires, qui mentionnent un emploi concessif pour alors que mais non pas pour tandis que.

\subsection{Catégorisation des correspondants de traduction}

Afin de pouvoir répondre à la question de savoir si la relation adversative ou concessive est explicitée dans la traduction de ces marqueurs du français en suédois, nous proposons une analyse des correspondants de traduction du tableau 3 repartis en trois catégories, qui se distinguent par le degré d'explicitation de la relation adversative ou concessive qu'implique le correspondant de traduction. Cette catégorisation est basée sur la conception de ces marqueurs qui peuvent marquer aussi bien la concomitance que le contraste ou la concession comme ambigus et moins explicites que les marqueurs adversatifs et concessifs «purs» (comme däremot dans (10) et fast dans (11)), conception qui peut évidemment être discutée.

La catégorie 1 englobe les occurrences où le degré d'explicitation de la relation adversative/concessive est considéré comme élevé dans le texte cible par rapport au texte source. Cette première catégorie se manifeste dans les cas où, comme dans (10) et (11), il y a un marqueur temporel/adversatif (alors que) dans le texte source, qui est traduit dans le texte cible par un marqueur adversatif/concessif (trots att) :

(12) Moi je voyais l'heure qui tournait et No qui ne se réveillait pas alors qu'elle avait son rendez-vous avec l'assistante sociale.

Jag såg att klockan gick och att No inte gick upp trots att hon hade en tid hos socialassistenten. (de Vigan)

Dans la deuxième catégorie, le degré d'explicitation de la relation adversative/concessive est égal en texte source et en texte cible. C'est par exemple le cas dans les occurrences où il y a un marqueur temporel/adversatif comme alors que dans le texte source qui est également traduit par un marqueur temporel/adversatif comme medan dans le texte cible, comme dans (13) :

(13) Mais le renard arctique seulement en couverture, alors que pour le roux, il nous reste couverture et manteau.

Men vi har bara fällar av polarräv medan det finns både fällar och kappor av rödräv. (Rambach) 
La catégorie 3, finalement, se compose des occurrences de degré d'explicitation de la relation adversative/concessive réduit dans le texte cible par rapport au texte source: nous trouvons dans le texte source un marqueur temporel/adversatif comme tandis que, qui n'a pas de correspondant de traduction explicite dans le texte cible (14).

(14) Elle en était à la cinquième foulée, quand son pied buta contre un appareil photo, pulvérisant le flash, tandis que l'autre pied écrasait la main inerte de Lee Song. På femte klivet stötte hon till en kamera med ena foten så att blixten utlöstes. Den andra foten trampade på Lee Songs orörliga hand. (Rambach)

Dans cette troisième catégorie, où le texte cible n'offre pas de correspondant explicite au marqueur du texte source, le lien, qu'il soit temporel, adversatif ou concessif, est sousentendu dans le texte cible.

Pour les cas de traduction par un marqueur qui n'est ni temporel, ni adversatif ni concessif, mais par exemple causal, nous avons également recours à une quatrième catégorie qui couvre ces cas autres que les marqueurs qui peuvent marquer la temporalité, le contraste ou la concession.

L'analyse de toutes les occurrences de alors que et tandis que dans les deux corpus répertoriés en ces quatre catégories de types de correspondants de traduction montre, sans grande surprise, que la plupart des occurrences sont traduites par un marqueur qui peut être catégorisé comme relevant du même degré d'explicitation de la relation adversative ou concessive (voir figure 1). C'est-à-dire que la plupart des occurrences de alors que et de tandis que sont, comme dans (8), (9) et (13), traduites par medan ou samtidigt som, qui peuvent également marquer aussi bien la concomitance que le contraste ou la concession, ou bien par un marqueur clairement temporel comme då ('alors') ou när ('quand'), aussi analysés comme relevant de la catégorie 2 .

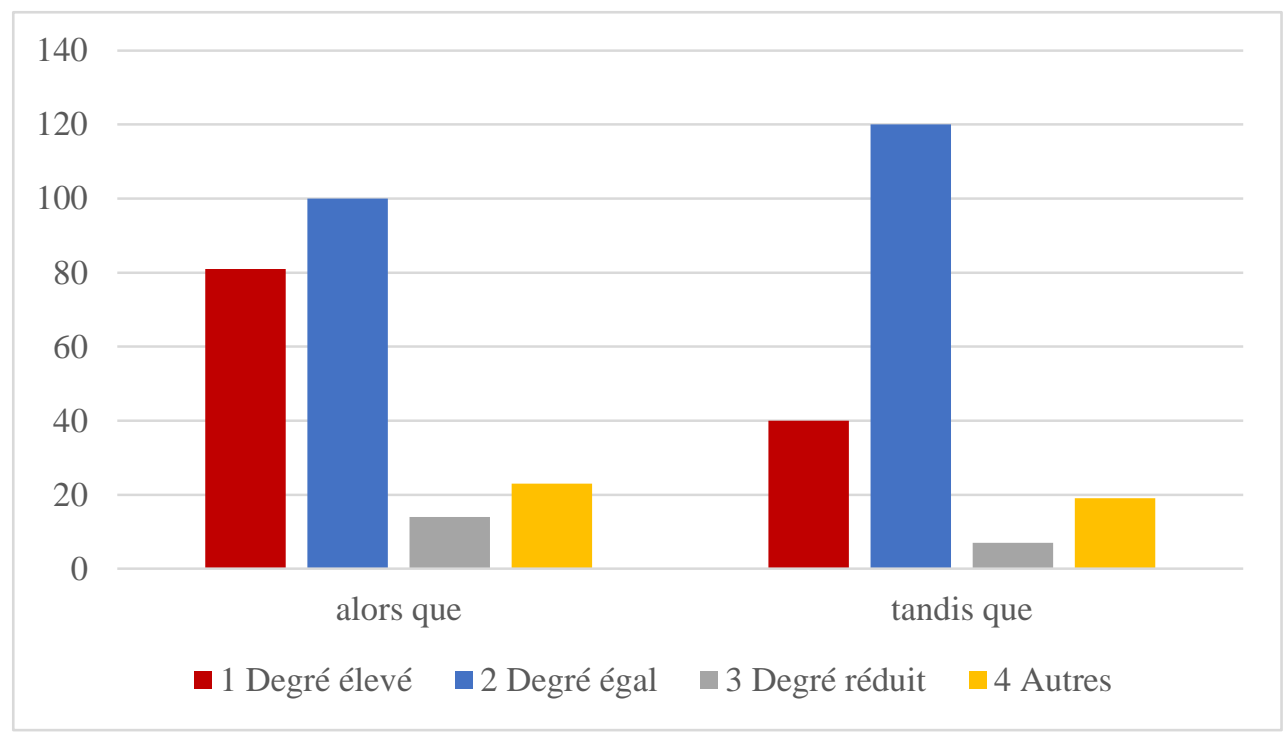

Figure 1 : Nombre d'occurrences de alors que et de tandis que des deux corpus traduits par un type de traduction de catégorie $1,2,3$ ou 4.

Mais nous pouvons également constater que la colonne gauche, qui résume le nombre d'occurrences de traduction par catégorie 1, est assez élevée, et surtout dans le cas de alors que. Dans 81 des 220 occurrences de alors que (soit un tiers des occurrences), et dans plus 
d'un cinquième des occurrences de tandis que, 40 sur 184, le marqueur a été traduit par un marqueur clairement adversatif ou concessif.

\subsection{Marquage double}

Parmi ces cas de marquage de la relation adversative/concessive élevée dans le texte cible, il y a beaucoup de cas similaires aux exemples (10), (11) et (12), dans lesquels alors que ou tandis que est traduit par un seul marqueur adversatif ou concessif, mais surtout pour tandis que, le corpus comporte également une grande quantité de cas où la relation adversative ou concessive est explicitée dans le texte cible de manière double, ce qui se manifeste de deux manières différentes. Premièrement, il peut y avoir deux marqueurs qui sont tous les deux adversatifs ou concessifs :

(15) Alors que bien des analystes voyaient dans cette phase suprême de l'évolution industrielle l'annonce d'un mouvement plus radical, plus révolutionnaire que jamais, j'annonçais, dès le début des années 1960, le déclin de la conscience de classe ouvrière et donc du syndicalisme comme mouvement social.

Många analytiker såg i denna högsta fas av den industriella utvecklingen ett förebud om en rörelse som skulle vara mer radikal och revolutionär än någonsin förr. Men jag förutspådde däremot redan i början av 1960-talet att klasskänslan hos arbetarna var på tillbakagång, vilket också betydde en tillbakagång för fackföreningsrörelsen som social rörelse. (Touraine)

Dans (15), alors que du texte source est traduit aussi bien par men que par däremot, qui marquent tous les deux le contraste entre les deux propositions reliées.

Dans le deuxième cas de figure du marquage double, nous trouvons dans le texte cible un marqueur temporel/adversatif en combinaison avec un marqueur adversatif ou concessif :

(16) Ces deux décennies sont également celles où le pourcentage d'œuvres sans aucune référence au thème se situe le plus bas, autour de $22 \%$, alors qu'il atteint environ le double durant les deux précédentes et les deux suivantes et ne baisse que peu, à $33 \%$, de 1760 à 1769, s'établissant finalement ensuite au quart de la production, sans grande fluctuation jusqu'à la fin du siècle.

Under dessa två årtionden är antalet böcker som inte har någon hänvisning till djävulstemat som lägst, runt 22 procent, medan det däremot är dubbelt så stort under de två föregående och de två 256 kommande årtiondena, och det sjunker endast något, till 33 procent från 1760 till 1769, och slutligen utgör det en fjärdedel av alla böcker utan större variationer ända fram till århundradets slut. (Muchembled)

Dans (16), le contraste entre les propositions, marqué par alors que dans le texte source, est dans le texte cible marqué aussi bien par medan que par däremot, où ce dernier est un marqueur clairement adversatif.

Si l'on recense les occurrences qui témoignent d'un marquage double de la relation en traduction du français en suédois, nous constatons, comme il ressort de tableau 4, que ces occurrences représentent la plupart des cas de la catégorie 1, de marquage élevé, de tandis que, mais que alors que est moins souvent traduit par une telle construction double. 


\begin{tabular}{|c|c|c|}
\hline & alors que & tandis que \\
\hline Traduction par marquage double & 14 & 25 \\
\hline $\begin{array}{l}\text { Autres occurrences de la catégorie } 1 \\
\text { (un marqueur adversatif ou concessif) }\end{array}$ & 67 & 15 \\
\hline $\begin{array}{l}\text { Nombre total d'occurrences de la } \\
\text { catégorie } 1\end{array}$ & 81 & 40 \\
\hline
\end{tabular}

Tableau 4 : Nombre d'occurrences de alors que et de tandis que des textes source français traduites par un seul marqueur adversatif ou concessif ou par un double marqueur.

Nous rappelons que Teleman et al. (1999) mentionne que la relation adversative ou concessive peut être doublement soulignée : par un marqueur adversatif, comme däremot, ou concessif, comme ändå, suivant medan ou samtidigt som. Mais, l'analyse des occurrences de medan et de samtidigt som dans les textes source suédois de nos corpus nous permet de constater que ce marquage double y est très rare : des 287 occurrences recensées de medan, seulement cinq sont suivies un tel marqueur, et aucune des 71 occurrences de samtidigt som des textes source suédois ne représente un tel marquage double.

\subsection{Tendance générale de traductions?}

Il faut prendre en compte la possibilité que la tendance d'explicitation dans la traduction puisse s'expliquer par la tendance générale d'explicitation lors de toute traduction, proposée notamment par Blum-Kulka (1986). Mais les analyses de la traduction dans l'autre sens indiquent le contraire; nous constatons en effet qu'il n'y a que des cas très rares de traduction de medan ou de samtidigt som par un marqueur plus explicite en français. Sur les 287 occurrences de medan et les 71 occurrences de samtidigt som, il n'y a que trois occurrences où medan est traduit de manière plus explicite et deux occurrences de samtidigt som où c'est le cas. Parmi les occurrences de medan dans les textes source suédois, 24 peuvent être catégorisées comme appartenant à la catégorie du degré d'explicitation réduit, contre 10 pour samtidigt som. Même si dans la grande majorité des occurrences des deux marqueurs le correspondant de traduction implique une explicitation de degré égal, il y a plutôt implicitation qu'explicitation de la relation dans cette direction de traduction.

Le fait que la traduction des marqueurs suédois en français ne montre pas la même tendance d'explicitation que celle de la traduction de alors que et de tandis que en suédois ne permet pas forcément d'annuler l'explication qu'il s'agirait dans la traduction du français en suédois d'un exemple de caractéristique typique de la traduction, puisque comme l'affirment aussi bien Nome (2013) que Engel et Sullet-Nylander (2015), il est possible que les différentes directions de traductions entre ces deux langues spécifiques soient réglées par des normes différentes, ce qui pourrait expliquer que l'explicitation ne se révèle que dans une des directions de la traduction. Étant donné que la tendance d'explicitation du lien adversatif et concessif est si forte dans la traduction en suédois de ces marqueurs dans nos données, mais ne se manifeste pas dans la traduction en français, il nous semble pourtant légitime de supposer que la différence s'explique aussi par une différence sémantique entre les marqueurs des deux langues. Il se peut que la tendance à explicitation s'explique par une différence de contrainte de simultanéité pour les marqueurs de chaque langue. 


\section{Temps verbaux différents des propositions reliées}

Même si l'emploi de temps verbaux identiques dans les propositions reliées par la conjonction n'implique pas forcément que les actions sont simultanées, il est intéressant de constater que dans l'analyse des occurrences des textes source, nous avons pu relever une différence entre les marqueurs étudiés concernant la fréquence d'occurrences où différents temps verbaux sont employés dans la proposition principale et la proposition subordonnée, comme dans (17) :

(17) La politique est soumise à l'éthique, alors que pendant longtemps elle a voulu se constituer elle-même en éthique, en morale civique ou en défense d'un avenir radieux contre le passé. (Touraine)

Sans compter les occurrences françaises d'emploi des temps verbaux différents au passé, qui peuvent relever d'une différence aspectuelle, nous constatons que alors que relie dans $27 \%$ des occurrences des propositions avec des temps verbaux différents. Pour tandis que, seulement $7 \%$ des occurrences présentent des temps verbaux différents dans les deux propositions. Pour les marqueurs suédois, l'emploi de temps verbaux différents est rare : 5\% des occurrences de medan (14 occurrences) et une seule occurrence de samtidigt som.

Dans les 14 cas de medan et la seule occurrence de samtidigt som de temps verbaux différents, il s'agit dans presque tous les cas d'un parfait ou d'un plus-que-parfait, désignant le résultat de l'action, ce qui laisse supposer que c'est plutôt un état qui est décrit, qui est simultané avec l'action décrite dans l'autre proposition, au présent dans (18) :

(18) Han som sitter på motorhuven har rakat bort allt hår, medan den andre har en frisyr som en skolpojke.

Celui qui est assis sur le capot est complètement rasé, l'autre est coiffé comme un écolier. (Kepler)

On ne peut guère dire que les actions dans ce cas se situent ou se déroulent à des moments différents, mais c'est plutôt la validité simultanée du résultat de la première action avec la deuxième qui est soulignée. Ceci est certes aussi le cas dans plusieurs occurrences des marqueurs français qui apparaissent dans des propositions dont les verbes sont conjugués dans différents temps, mais les cas où les actions ou leur résultat sont clairement non simultanés ne sont toutefois pas rares.

Ce résultat nous permet de questionner l'affirmation de Teleman et al. (1999) concernant les marqueurs suédois, selon laquelle la proposition subordonnée ne doit pas nécessairement exprimer une action simultanée. Cette hypothèse devrait néanmoins être examinée dans des données de corpus plus vastes. L'éventuelle difficulté de relier des actions qui ne sont pas simultanées pourrait pourtant expliquer la tendance à explicitation de la relation adversative ou concessive dans les cas où un autre marqueur, ou un marqueur double, rend cette relation explicite dans le texte cible suédois.

Sans que cela explique la totalité des cas d'explicitation de la relation adversative ou concessive que nos données fournissent, nous constatons que lorsqu'il y a deux temps verbaux différents en français, on a souvent recours à un marqueur de degré d'explicitation élevé en suédois, comme dans (17), où c'est le marqueur men ('mais') qui relie les deux propositions : 
(17) La politique est soumise à l'éthique, alors que pendant longtemps elle a voulu se constituer elle-même en éthique, en morale civique ou en défense d'un avenir radieux contre le passé. (Touraine)

Under en lång tid försökte politiken etablera sig själv som en etik eller som en civil moral och den ville försvara den strålande framtiden mot en förgången tid. Men nu har politiken underordnats etiken. (Touraine)

Dans la plupart des cas où deux temps verbaux différents sont employés en français, sans que le degré d'explicitation ne soit élevé dans la traduction, il y a une autre modification du contexte, à savoir notamment un changement des temps verbaux lors de la traduction, de façon que soit employé le même temps verbal dans les deux propositions du texte cible suédois :

(19) La société américaine de la fin du $\mathrm{XX}^{\mathrm{e}}$ siècle porte imprégnée au cœur même de son tissu la hantise du démon, alors que celle-ci s'est fortement atténuée de l'autre côté de l'Atlantique.

Det amerikanska samhället vid 1900-talets slut har djävulsskräcken djupt invävd i sin stomme medan den kraftigt minskar på andra sidan Atlanten. (Muchembled)

Ainsi, dans (19), le passé composé de la subordonnée du texte source a été traduit par un verbe au présent, temps verbal que l'on retrouve également dans la proposition principale.

\section{Conclusion}

L'objectif de cette étude était d'identifier des tendances de la traduction des marqueurs de simultanéité, de contraste et de concession dans la traduction du français en suédois. Nous avons pu constater une tendance à explicitation de la relation adversative ou concessive lors de la traduction de tandis que, et surtout dans celle de alors que, au moyen d'une traduction par un marqueur adversatif ou concessif. Dans la traduction de tandis que, medan est souvent renforcé par un marqueur adversatif. Ce marquage double de la relation adversative ou concessive dans l'emploi de medan ou samtidigt som est un trait caractéristique des textes cibles suédois de nos données, alors qu'il est très rare dans les textes source suédois. Ce résultat pourrait indiquer que les marqueurs suédois ne peuvent pas toujours marquer à eux seuls le contraste ou la concession.

Il semble en effet que l'emploi adversatif de medan et de samtidigt som soit plus contraint que celui de tandis que et surtout que celui de alors que, notamment en ce qui concerne la simultanéité entre les actions. Nous avons pu constater que l'emploi de temps verbaux différents dans la subordonnée et la principale est plus fréquent pour les marqueurs français, surtout pour alors que, ce qui pourrait indiquer que ceux-ci peuvent être employés plus que leurs correspondants suédois hors contexte de concomitance, correspondant à leur valeur source. Ceci pourrait aussi indiquer que les marqueurs français, et surtout alors que, ont passé à un stade plus avancé, au stade de contexte de passage, dans le processus de grammaticalisation, tandis que les marqueurs suédois se trouvent dans un stade de contexte de transition. De plus vastes études comparatives seront nécessaires pour le confirmer. Il apparaît toutefois que la traduction par uniquement medan ou samtidigt som semble être évitée dans des contextes où il est difficile d'interpréter les deux actions reliées comme simultanées, ce qui pourrait indiquer que la contrainte de simultanéité est plus forte pour les marqueurs 
suédois, et que leur valeur adversative ou concessive n'est pas encore conventionnalisée au même degré que celle des marqueurs français.

\section{Bibliographie}

Bat-Zeev Shyldkrot, Hava. 1987. "Quand, lorsque et tandis que: un cas classique d'évolution sémantique ». Romance Notes 28(1) : 45-50.

Blum-Kulka, Shoshana. 1986. «Shifts of Cohesion and Coherence in Translation». In Interlingual and Intercultural Communication. Discourse and Cognition in Translation and Second Language Acquisition Studies, édité par Juliane House et Shoshana Blum-Kulka, 17-35. Tübingen: Gunter Narr Verlag.

Dictionnaire Larousse, http://www.larousse.fr/. [consulté le 29.01.18]

Engel, Hugues, et Françoise Sullet-Nylander. 2015. «Relations argumentatives en traduction : les connecteurs causaux dans un corpus de textes à teneur scientifique ». Synergies Pays Scandinaves 10 : 23-38.

Le Grand Robert de la langue française, 1986. $2^{\mathrm{e}}$ éd. éditée par Alain Rey. Paris : Les dictionnaires Robert.

Heine, Bernd. 2002. « On the role of context in grammaticalization ». In New Reflections on Grammiticalization, édité par Ilse Wischer et Gabriele Diewald, 83-101. Amsterdam: John Benjamins.

Hellqvist, Birgitta. 2015. Le gérondif en français et les structures correspondantes en suédois. Etude contrastive. Uppsala : Acta Universitatis Upsaliensis.

Marchello-Nizia, Christiane. 2006. Grammaticalisation et changement linguistique. Bruxelles : Duculot.

Morel, Mary-Annick. 1996. La concession en français. Paris : Ophrys.

Nationalencyclopediens ordbok, https://www.ne.se/ordb\%C3\%B6cker/\#/dictionary/neordbok-sv-sv [consulté le 29.01.18]

Le Nouveau Petit Robert. 1993. Edité par Josette Rey-Debove et Alain Rey. Paris : Dictionnaires Le Robert.

Nome, Astrid. 2013. Connectives in Translation: Explicitation and Relevance. Universitetet $\mathrm{i}$ Oslo.

Rey, Alain. 1998. Dictionnaire historique de la langue française. Paris : Dictionnaires Le Robert.

SAOB : Svenska Akademiens Ordbok. 1943. https://www.saob.se/ [consulté le 29.01.18]

Stage, Lilian. 1991. "Analyse syntaxique et sémantique de la conjonction dans les propositions factuelles ». Revue romane 26 (2) : 163-205.

Svensson, Maria. 2010. Marqueurs corrélatifs en français et en suédois. Étude sémanticofonctionnelle de d'une part... d'autre part, d'un côté... de l'autre et de non seulement... mais en contraste. Uppsala : Acta Universitatis Upsaliensis.

Teleman, Ulf, Staffan Hellberg et Erik Andersson. 1999. Svenska Akademiens Grammatik 4 Satser och meningar. Stockholm: Svenska Akademien. 